\title{
Perturbations of stealth black holes in degenerate higher-order scalar-tensor theories
}

\author{
Claudia de Rham $\oplus^{1,2, *}$ and Jun Zhang $\oplus^{1, \dagger}$ \\ ${ }^{1}$ Theoretical Physics, Blackett Laboratory, Imperial College, London SW7 2AZ, United Kingdom \\ ${ }^{2}$ Centre for Education and Research in Cosmology and Astrophysics, Department of Physics, \\ Case Western Reserve University, 10900 Euclid Ave, Cleveland, Ohio 44106, USA
}

(Received 28 August 2019; published 9 December 2019)

\begin{abstract}
Among the scalar-tensor modified theories of gravity, degenerate higher-order scalar-tensor (DHOST) models could play a special role for dark energy while being consistent with current observations, notably those constraining the speed of gravitational waves. Schwarzschild-de Sitter black holes were shown to be exact solutions of a particular subclass of quadratic DHOST theories, while carrying a nontrivial scalar profile that linearly evolves in time and hence potentially providing exciting new phenomenological windows to explore this model. We investigate the physical perturbations about such black holes and find that the odd-parity tensor perturbations behave in a way indistinguishable to general relativity. On the other hand, the effective metric for the (even-parity) scalar perturbations is singular, indicating that those exact black hole solutions are infinitely strongly coupled and cannot be trusted within the regime of validity of the DHOST effective field theory. We show how this strong coupling result is generalizable to a whole class of solutions with arbitrary manifolds both for DHOST and Horndeski.
\end{abstract}

DOI: $10.1103 /$ PhysRevD.100.124023

\section{INTRODUCTION}

The discovery of the cosmic accelerated expansion has motivated numerous studies on modifications of gravity in the infrared. The uniqueness of general relativity (GR) implies that any model of model gravity necessarily needs to involve additional degrees of freedom (d.o.f.) or a breaking of locality or Lorentz invariance. Including a scalar field and exploring scalar-tensor theories is therefore one of the most natural and minimalistic way to gravity beyond GR. Usually, scalar-tensor theories are considered as effective theories of more fundamental theories and provide a general framework to explain the observed cosmic acceleration phenomenologically. Under this consideration, many attempts have been made to construct the most generic consistent theory that propagates only one scalar d.o.f. while interacting with gravity. For example, Horndeski theories [1,2] are constructed as the most general scalar-tensor theory in four-dimensional spacetime yielding only second order field equations. In theories with multiple fields, higher-order field equations can still

\footnotetext{
*c.de-rham@imperial.ac.uk

jun.zhang@imperial.ac.uk
}

Published by the American Physical Society under the terms of the Creative Commons Attribution 4.0 International license. Further distribution of this work must maintain attribution to the author(s) and the published article's title, journal citation, and DOI. Funded by SCOAP ${ }^{3}$. propagate a single d.o.f. if their Lagrangian is degenerate $[3,4]$, and this argument was used to further extend scalartensor theories to a more general class of degenerate higherorder scalar-tensor (DHOST) theories in [5] (see [6-12] for related discussions and [13] for a review).

On the other hand, the direct detections of gravitational waves (GWs) from binary black hole and neutron star mergers made significant effects on our understanding of gravity. Particularly, the observation of GW170817 [14] together with its optical counterpart GRB170817A [15] constraints the speed difference between GWs and light (propagating on a cosmological background) down to $10^{-15}$ [16], which drastically restricts the viable candidates of scalar-tensor theories [17-21], provided that such scalartensor theories are still valid up to the LIGO frequency [22].

The development of the GW astronomy also stimulates the studies on black hole solutions in scalar-tensor theories, among which black holes with nontrivial scalar profile are of particular interests. No-hair theorem has been proved for the shift-symmetric Horndeski theory [23] and for the shift-symmetric Gleyzes-Langlois-Piazza-Vernizzi (GLPV) theory [24], which is a subclass of DHOST theories. Such theorems state that if the coupling functions of the theories are regular, the static, spherically symmetric, and asymptotically flat black hole solutions with static scalar field must have the Schwarzschild metric and the constant scalar field. Hairy black holes are allowed if some of the conditions are violated. For example, by violating the regularity condition, there are asymptotically flat hairy 
black holes in the shift-symmetric Horndeski theory $[25,26]$ and in the shift-symmetric GLPV theory [24]. However, these solutions usually present metrics different from GR black holes. Another example is the solution found in the shift-symmetric GLPV theories that do not have the canonical kinetic term [24], which also circumvents the no-hair theorem. Also see [27-29] for hairy black holes in Einstein-Scalar-Gauss-Bonnet theories.

Hairy black hole solutions can also be found if one allows for time-dependence. ${ }^{1}$ For instance, within the context of shift-symmetric DHOST theories, a branch of hairy black holes is constructed by considering a linearly time-dependent scalar field profile $\varphi(t, r)=q t+\psi(r)$ and a constant kinetic term $X=\partial_{a} \varphi \partial^{a} \varphi$. The linearly timedependent part of the scalar field can be thought as the background field that is responsible for the cosmic acceleration. Such solutions are investigated in [24,33-40]. Moreover, these solutions can be "stealth," which means that the nontrivial scalar hair does not gravitate at the background level. Stealth black hole solutions were initially introduced in [41]. Recently, the stealth linearly time-dependent solutions have been studied in the context of quadratic DHOST theories $[42,43]$. Taking a bottom up approach, these studies identify the theories that possess stealth Schwarzschild-de Sitter (SdS) black hole solutions.

Motivated by this wealth of fruitful solutions, the stability of physical implications of those solutions was rapidly explored. If the scalar field couples directly to matter sources, one would expect binary systems to radiate scalar gravitational waves which would typically be in tension with observations unless a Vainshtein mechanism or other type of screening is implemented [44-49]. Moreover, in DHOST theories, even when the scalar mode may not a priori directly couple to external matter sources, since the physical propagating d.o.f. are mixed between the metric and the covariant scalar, implicit matter coupling typically appears in such theories which can then also lead to gravitational scalar radiation.

As for the stability of such solutions, for shift-symmetric Horndeski theories it was initially argued in [50] that they could be unstable against odd-parity perturbation; however, this statement was more recently revisited in [51,52]. For specific time slicings, it was argued in [50] that the positivity of either the kinetic or radial gradient energy would be violated in the vicinity of the horizon. However, that particular statement is gauge dependent and in [51,52] it was shown how there could exist a slicing for which the theory was stable everywhere. Even if the kinetic or the gradient term where to switch sign in all slicings, this would only signal the breakdown of the Horndeski effective field theory when either the kinetic or the gradient term

\footnotetext{
${ }^{1}$ This point is also related to the existence of black hole solutions $[30,31]$ in other models of modified gravity such as massive gravity [32].
}

becomes sufficiently small and the predictability of the Horndeski effective field theory therefore fails before the instability can even occur (see [53] for a related discussion).

In this study, we will explore the stability and validity (in the EFT sense) of the exact quadratic DHOST solution found in $[42,43]$. Odd perturbations have already been the subject of a very interesting analysis [40] (which appeared during the final stages of this work), and in the rest of this paper we shall investigate both the odd and even-parity perturbations about the stealth black hole solutions in the shift-symmetric quadratic DHOST theories. In agreement with [40], we show that such solutions are stable against odd-parity perturbations. Indeed, the odd-parity perturbations are exactly the same as that of GR black holes. The even-parity perturbations however differ due to the presence of the scalar field and while the diagonalized would-be tensor modes could a priori be stable, the diagonalized scalar mode sees a singular effective metric, indicating the stealth black hole solution in the shift-symmetric quadratic DHOST theories can unfortunately not be trusted.

To put the current findings in perspective, we highlight that the nature of this problem is more severe than that observed in [50] for Horndeski black holes. Indeed, fluctuations about the shift-symmetric Horndeski black hole solutions are well-behaved at sufficiently large distances and the theory only becomes unreliable close to the horizon (where either the kinetic or gradient term becomes small, before they would become negative). Such solutions can therefore still potentially provide relevant phenomenology away from the horizon. For the stealth black hole solutions in the shift-symmetric quadratic DHOST theories on the other hand, the effective scalar fluctuations are always everywhere and all the time degenerate and those solutions can therefore never and nowhere be trusted. These types of pathologies are very similar in nature to those observed about the exact static black solutions found in massive gravity [31], where it was shown that solutions that perfectly mimic GR black hole solutions, actually do so by effectively suppressing the graviton mass on those backgrounds and therefore making the additional d.o.f. present in massive gravity infinitely strongly coupled. The case of DHOST appears to be very analogous to the case of massive gravity: black hole solutions can only precisely be the same as in GR if the effect of the scalar field is effectively entirely suppressed, and therefore making this d.o.f. infinitely strongly coupled. Instead a perhaps more promising direction for black hole solutions in DHOST theories just like in massive gravity is the presence of a (small but nonetheless nonzero) time dependence of the metric. This time dependence would typically be governed by the graviton mass scale in massive gravity or the dark-energy scale in DHOST theories and could therefore 
imply a time-evolution only visible on timescales of the order of the age of the Universe. ${ }^{2}$

The rest of the paper is organized as follows. In Sec. II, we first present the shift-symmetric quadratic DHOST theories and review the linear time-dependent black hole solutions. In Sec. III, we derive equations of both odd- and even-parity perturbations and highlight the presence of an (infinitely) strongly coupled d.o.f. The strong coupling issue is then generalized to a larger class of solutions with generic manifolds in DHOST theories including rotating black hole solutions in Sec. IV. The implications for a class of solutions in Horndeski is also highlighted. Section V is then conclusions and outlook.

\section{STEALTH BLACK HOLES IN SHIFT- SYMMETRIC DHOST THEORIES}

The Lagrangian of the shift-symmetric DHOST theory up to quadratic order is given by [5]

$$
\mathcal{L}_{\mathrm{qDHOST}}=P(X)+Q(X) \square \varphi+F(X) R+\sum_{i=1}^{5} A_{i}(X) B_{i}
$$

where

$$
\begin{aligned}
& \quad B_{1}=\varphi_{a b} \varphi^{a b}, \quad B_{2}=(\square \varphi)^{2}, \\
& B_{3}=\varphi^{a} \varphi^{b} \varphi_{a b} \square \varphi, \quad B_{4}=\varphi^{a} \varphi_{a b} \varphi^{b c} \varphi_{c}, \\
& B_{5}=\left(\varphi_{a b} \varphi^{a} \varphi^{b}\right)^{2},
\end{aligned}
$$

with $\varphi_{a}=\partial_{a} \varphi, \varphi_{a b}=D_{a} D_{b} \varphi$ and $X=\varphi_{a} \varphi^{a}=g^{a b} \varphi_{a} \varphi_{b}$. In the wake of GW170817 [21], the requirement that the speed of GWs should be the same as light in a cosmological background imposes the following conditions (if one were to assume that the DHOST effective field theory remained sufficiently under control at LIGO frequency scale [22]),

$$
\begin{aligned}
& A_{1}=A_{2}=0, \\
& A_{4}=\frac{1}{8 F}\left[48 F^{\prime 2}-8\left(F-X F^{\prime}\right) A_{3}-X^{2} A_{3}^{2}\right], \\
& A_{5}=\frac{1}{2 F}\left(4 F^{\prime}+X A_{3}\right) A_{3},
\end{aligned}
$$

where here a prime denotes the derivative with respect to the argument $X$. In addition, it also requires $A_{3}=0$ to prevent a rapid decay of GWs into the scalar field [55] (see also [56]). This condition as well as the condition $A_{1}=0$ was not imposed in the recent analysis presented in [40].

Thus, the subclass of DHOST theories that will be considered in this paper is

\footnotetext{
${ }^{2}$ This point is also tightly linked to the need of small yet nonvanishing amount of space-dependence for consistent massive cosmology solutions [54].
}

$$
\begin{aligned}
S= & \int \mathrm{d}^{4} x \sqrt{-g}[P(X)+Q(X) \square \varphi+F(X) R \\
& \left.+\frac{6 F^{2}}{F} \varphi_{a b}^{2} \varphi^{a} \varphi^{b}+\mathcal{L}^{(\text {matter })}\left(g, \psi_{i}\right)\right],
\end{aligned}
$$

where we used $A_{4}=6 F^{\prime 2} / F$. Note that we have also included external matter fields $\psi_{i}$ that only couple to the metric $g_{\mu \nu}$. Even though at this level there is no direct coupling between $\varphi$ and the external matter sources, we will still see in what follows that the physical scalar d.o.f. in this theory does directly couple (already at tree-level) to external sources. This is due to the nontrivial mixing of the physical d.o.f. in these types of degenerate field theories.

For the back hole solutions, we assume the ansatz,

$$
\begin{aligned}
& \mathrm{d} s^{2}=\bar{g}_{a b} \mathrm{~d} x^{a} \mathrm{~d} x^{b}=-A(r) \mathrm{d} t^{2}+\frac{1}{A(r)} \mathrm{d} r^{2}+r^{2} \mathrm{~d} \Omega^{2}, \\
& \quad \text { and } \bar{\varphi}=q t+\psi(r),
\end{aligned}
$$

where a bar refers to the background. Note that $A(r)$ has nothing to do with the $A_{i}(X)$ previously introduced in (2.1). We further require $X$ to be a constant, $X=X_{0}$, which implies [33]

$$
\frac{d \psi}{d r}= \pm \frac{1}{A} \sqrt{q^{2}+X_{0} A}
$$

Under the ingoing Eddington-Finkelstein coordinates $(v, r)$ defined by $\mathrm{d} v=\mathrm{d} t+\mathrm{d} r / A$, it can be shown that $\varphi \simeq q v$ at the vicinity of the future event horizon $[33,43]$. Therefore, we will pick the branch with the "+" sign, in which case $\varphi$ is regular at the future event horizon. In this paper, we will mostly focus on $\mathrm{SdS}$ black holes, i.e.,

$$
A(r)=1-\frac{2 M}{r}-\Lambda r^{2}
$$

The theories also allow Schwarzschild black holes with linear time dependence, which can be obtained by sending $\Lambda \rightarrow 0$ [43], and our analysis applies as well. Following the analysis presented in $[42,43]$, we can see that the ansatz (2.6) solves the equation of motions in two following cases:

Case 1: $X_{0}=-q^{2}$,

$$
P+6 \Lambda F=2 P^{\prime}+24 \Lambda F^{\prime}-9 \Lambda q^{2} A_{3}=Q^{\prime}=0,
$$

Case 2: $X_{0} \neq-q^{2}$,

$$
P+6 \Lambda F=P^{\prime}+12 \Lambda F^{\prime}=Q^{\prime}=A_{3}=0,
$$

where all terms are evaluated at $X=X_{0}$. For theories with $A_{3}=0$, i.e., theories have no significant GWs to scalar field decay, the conditions of having black holes in those two cases are degenerate.

We point out that the model considered at the moment is equivalent to Horndeski after field redefinitions; however, we perform the analysis on perturbations in the DHOST 
frame as it allows us to easily generalize it to a more generic class of DHOST theories in what follows.

\section{BLACK HOLE PERTURBATIONS}

We first start with the covariant equations of motion. Varying the action (2.5) with respect to the inverse metric $g^{a b}$, we obtain the modified Einstein equation,

$$
\begin{aligned}
\mathcal{E}_{a b}= & F G_{a b}+F^{\prime}\left(R \varphi_{a} \varphi_{b}-D_{a} D_{b} X+\square X g_{a b}\right) \\
& -F^{\prime \prime}\left(X_{a} X_{b}-(\partial X)^{2} g_{a b}\right)+P^{\prime} \varphi_{a} \varphi_{b}-\frac{1}{2} P g_{a b} \\
& -\frac{1}{2} Q^{\prime}\left(X_{a} \varphi_{b}+\varphi_{a} X_{b}-X_{c} \varphi^{c} g_{a b}-2 \square \varphi \varphi_{a} \varphi_{b}\right) \\
& -\frac{1}{8} A_{4}\left(4 \square X \varphi_{a} \varphi_{b}-2 X_{a} X_{b}+(\partial X)^{2} g_{a b}\right) \\
& -\frac{1}{4} A_{4}^{\prime}(\partial X)^{2} \varphi_{a} \varphi_{b}-T_{a b}=0,
\end{aligned}
$$

where we define $X_{a} \equiv \partial_{a} X$. Equation (3.1) involves terms with three derivatives acting on $\varphi$. Here $T_{a b}$ is the (conserved) stress-energy tensor associated with the matter fields $\psi_{i}$. Conservation of $T_{a b}$, i.e., $\mathrm{D}_{a} T^{a}{ }_{b}=0$ sets the equation of motion for the matter fields $\psi_{i}$. Varying with respect to $\varphi$ yields the equation of motion for the scalar field

$$
\begin{aligned}
\mathcal{E}_{\varphi}= & D_{a}\left[Q^{\prime} X^{a}-2\left(F^{\prime} R+P^{\prime}+Q^{\prime} \square \varphi\right.\right. \\
& \left.\left.-\frac{1}{2} A_{4} \square X-\frac{1}{4} A_{4}^{\prime}(\partial X)^{2}\right) \varphi^{a}\right]=0 .
\end{aligned}
$$

To perturb the equations about the black hole solution, we write $g_{a b}=\bar{g}_{a b}+h_{a b}, \varphi=\bar{\varphi}+\delta \varphi$, and $T_{a b}=0+\delta T_{a b}$, where a subscript ${ }_{0}$ or a bar refers to the background. ${ }^{3}$ For convenience, we also keep $X=X_{0}+\delta X$ with $\delta X=$ $2 \bar{\varphi}^{a} \delta \varphi_{a}-\bar{\varphi}^{a} \bar{\varphi}^{b} h_{a b}$ where indices are raised and lowered with respect to the background $\operatorname{SdS}$ metric $\bar{g}_{a b}$. Working to first order in perturbations about the background, we then have the perturbed equations

$$
\begin{aligned}
\mathcal{E}_{a b}^{(1)}= & F_{0} \delta G_{a b}+3 \Lambda F_{0} h_{a b}+F_{0}^{\prime} \bar{\varphi}_{a} \bar{\varphi}_{b} \delta R-\delta T_{a b} \\
& +\left[3 \Lambda F_{0}^{\prime} \bar{g}_{a b}+\left(F_{0}^{\prime \prime} R_{0}+P_{0}^{\prime \prime}+Q_{0}^{\prime \prime} \square \bar{\varphi}\right) \bar{\varphi}_{a} \bar{\varphi}_{b}\right] \delta X \\
& -F_{0}^{\prime} \delta X_{a b}+\left(F_{0}^{\prime} \bar{g}_{a b}-\frac{3 F_{0}^{\prime 2}}{F_{0}} \bar{\varphi}_{a} \bar{\varphi}_{b}\right) \square \delta X=0
\end{aligned}
$$

and

$$
\begin{aligned}
\mathcal{E}_{\varphi}^{(1)}= & \mathrm{D}_{a}\left\{\overline { \varphi } ^ { a } \left[-2 F_{0}^{\prime} \delta R+\frac{6 F_{0}^{\prime 2}}{F_{0}} \square \delta X\right.\right. \\
& \left.\left.-2\left(F_{0}^{\prime \prime} R_{0}+P_{0}^{\prime \prime}+Q_{0}^{\prime \prime} \square \bar{\varphi}\right) \delta X\right]\right\}=0,
\end{aligned}
$$

where we have used conditions (2.9) or (2.10) and $R_{0}=12 \Lambda$, and have defined $\delta X_{a b}=D_{a} D_{b} \delta X$. To make further progress and properly separate out the relevant d.o.f., we consider the following linear combinations of the equations of motion:

$$
\left(F_{0}-F_{0}^{\prime} X_{0}\right) \mathcal{E}_{\varphi}^{(1)}-2 F_{0} \mathrm{D}_{a}\left(\mathcal{E}^{(1)} \varphi^{a}\right)=0
$$

and

$\mathcal{E}_{a b}^{(1)}-\frac{F_{0}^{\prime}}{F_{0}^{\prime} X_{0}-F_{0}} \bar{\varphi}_{a} \bar{\varphi}_{b} \mathcal{E}^{(1)}+\frac{1}{2} \frac{F_{0}}{F_{0}^{\prime} X_{0}-F_{0}} \bar{g}_{a b} \mathcal{E}^{(1)}=0$,

where we used the notation $\mathcal{E}^{(1)} \equiv \mathcal{E}^{(1) c}{ }_{c}=\bar{g}^{a b} \mathcal{E}_{a b}^{(1)}$, leading to

$$
\begin{aligned}
& \mathrm{D}_{a}\left[\overline { \varphi } ^ { a } \left(-2 F_{0}\left(F_{0}^{\prime \prime} R_{0}+P_{0}^{\prime \prime}+Q_{0}^{\prime \prime} \square \bar{\varphi}+2 \overline{A_{4}}\right) \delta X\right.\right. \\
& \left.\left.\quad+4 F_{0}^{\prime} \delta T\right)\right]=0
\end{aligned}
$$

and

$$
F_{0}\left(\delta R_{a b}-3 \Lambda h_{a b}\right)=F_{0} S_{a b},
$$

respectively. Here we have defined the source tensor $S_{a b}$ as

$$
\begin{aligned}
F_{0} S_{a b} \equiv & \delta T_{a b}+\frac{1}{F_{0}^{\prime} X_{0}-F_{0}}\left(\frac{1}{2} F_{0} \bar{g}_{a b}-F_{0}^{\prime} \bar{\varphi}_{a} \bar{\varphi}_{b}\right) \delta T+\frac{1}{2} F_{0}^{\prime} \bar{g}_{a b} \square \delta X+F_{0}^{\prime} \delta X_{a b} \\
& -\left[3 \Lambda F_{0}^{\prime}+\frac{6 \Lambda F_{0} F_{0}^{\prime}}{F_{0}^{\prime} X_{0}-F_{0}}+\frac{1}{2} \frac{F_{0} X_{0}}{F_{0}^{\prime} X_{0}-F_{0}}\left(F_{0}^{\prime \prime} R_{0}+P_{0}^{\prime \prime}+Q_{0}^{\prime \prime} \square \bar{\varphi}\right)\right] \bar{g}_{a b} \delta X \\
& +\left[\frac{12 \Lambda F_{0}^{\prime 2}}{F_{0}^{\prime} X_{0}-F_{0}}+\frac{F_{0}}{F_{0}^{\prime} X_{0}-F_{0}}\left(F_{0}^{\prime \prime} R_{0}+P_{0}^{\prime \prime}+Q_{0}^{\prime \prime} \square \bar{\varphi}\right)\right] \bar{\varphi}_{a} \bar{\varphi}_{b} \delta X .
\end{aligned}
$$

\footnotetext{
${ }^{3}$ To be technically correct, the background expression for $T_{a b}$ does not actually vanish everywhere, it is a delta function at the origin, scaling with $M$ and corresponding to the physical source of the back hole. However, away from the origin, the background part of the stress-energy tensor vanishes for the black hole situation considered here and is therefore irrelevant for the rest of this study.
} 
In the following, we shall decompose the perturbations based on their behaviors under parity transformations $(\theta, \phi) \rightarrow(\pi-\theta, \phi+\pi)$. This decomposition allows us to consider odd perturbation and even perturbation separately.

\section{A. Odd sector}

The odd-parity perturbation of the metric can be written as [57-59]

$$
h_{a b}^{\text {odd }}=\left(\begin{array}{cccc}
0 & 0 & -\mathrm{h}_{0} \csc \theta Y_{\ell m, \phi} & \mathrm{h}_{0} \sin \theta Y_{\ell m, \theta} \\
0 & 0 & -\mathrm{h}_{1} \csc \theta Y_{\ell m, \phi} & \mathrm{h}_{1} \sin \theta Y_{\ell m, \theta} \\
-\mathrm{h}_{0} \csc \theta Y_{\ell m, \phi} & -\mathrm{h}_{1} \csc \theta Y_{\ell m, \phi} & \frac{1}{2} \mathrm{~h}_{2} \csc \theta \mathcal{X} & -\frac{1}{2} \mathrm{~h}_{2} \sin \theta \mathcal{W} \\
\mathrm{h}_{0} \sin \theta Y_{\ell m, \theta} & \mathrm{h}_{1} \sin \theta Y_{\ell m, \theta} & -\frac{1}{2} \mathrm{~h}_{2} \sin \theta \mathcal{W} & -\frac{1}{2} \mathrm{~h}_{2} \sin \theta \mathcal{X}
\end{array}\right)
$$

where $\mathrm{h}_{0}, \mathrm{~h}_{1}, \mathrm{~h}_{2}$ are functions of $(t, r), Y_{\ell m}$ are the spherical Harmonics, a comma denotes the partial derivate, and

$$
\begin{aligned}
\mathcal{X} & =2\left(\partial_{\theta} \partial_{\phi}-\cot \theta \partial_{\phi}\right) Y_{\ell m}, \\
\mathcal{W} & =\left(\partial_{\theta} \partial_{\theta}-\cot \theta \partial_{\theta}-\csc ^{2} \theta \partial_{\phi} \partial_{\phi}\right) Y_{\ell m}
\end{aligned}
$$

Note that perturbation of the scalar field $\delta \varphi$ is even under the parity transformation, hence does not couple with $h_{a b}^{\text {odd }}$ and can be omitted in the odd perturbation equations. Moreover, explicate calculation shows that $h_{a b}^{\text {odd }} \varphi^{a} \varphi^{b}=0$ and hence $\delta X$ vanishes in the odd sector. Together with the fact that $\delta R^{\text {odd }}=0$, Eq. (3.3) simplifies to

$$
F_{0}\left(\delta G_{a b}+3 \Lambda h_{a b}\right)-\delta T_{a b}=0
$$

where $F_{0}$ plays the effective role of the Planck scale and we can therefore conclude that the odd-parity perturbation of the stealth SdS black holes considered here is identical to that in GR.

Relaxing the assumptions: The previous result relied on the assumption $A_{3}=0$ (so as to prevent GWs from decaying into dark energy); however, we may wonder what the effects would be if some of those assumptions were relaxed. Actually, we find that even in the case where $A_{3} \neq 0$ and hence $A_{5} \neq 0$, the odd sector of GWs still behave identically as in GR. We can see this by perturbing the Lagrangian to quadratic order and using the fact that $\bar{B}_{3}=\bar{B}_{5}=\delta^{(2)} B_{5}=0$, then we can see that the presence of $A_{3}$ and $A_{5}$ leads to two extra terms, $9 q^{2} \Lambda \bar{A}_{3} \bar{\varphi}^{a} \bar{\varphi}^{b} \bar{g}^{c d} h_{a c}^{\text {odd }} h_{b d}^{\text {odd }}$ and $\bar{A}_{3} \delta^{(2)} B_{3}$, which eventually cancel each other given the background solution. ${ }^{4}$

\section{B. Even sector}

Next we turn to the even-parity perturbations. The metric perturbations can be written as [57-59]

$$
h_{a b}^{\text {even }}=\left(\begin{array}{cccc}
A H_{0} & H_{1} & \mathcal{H}_{0} \partial_{\theta} & \mathcal{H}_{0} \partial_{\phi} \\
H_{1} & H_{2} / A & \mathcal{H}_{1} \partial_{\theta} & \mathcal{H}_{1} \partial_{\phi} \\
\mathcal{H}_{0} \partial_{\theta} & \mathcal{H}_{1} \partial_{\theta} & \mathcal{K}+\mathcal{G} \nabla_{\theta} \nabla_{\theta} & \mathcal{G} \nabla_{\theta} \nabla_{\phi} \\
\mathcal{H}_{0} \partial_{\phi} & \mathcal{H}_{1} \partial_{\phi} & \mathcal{G} \nabla_{\phi} \nabla_{\theta} & \sin ^{2} \theta \mathcal{K}+\mathcal{G} \nabla_{\phi} \nabla_{\phi}
\end{array}\right) Y_{\text {em }}
$$

where again $H_{0}, H_{1}, H_{2}, \mathcal{H}_{0}, \mathcal{H}_{1}, \mathcal{K}$, and $\mathcal{G}$ are functions of $(t, r)$, and $\nabla_{\theta, \phi}$ are covariant derivatives on the two-sphere of radius one. We now also have the scalar perturbation

$$
\delta \varphi=\Phi(t, r) Y_{\ell m}
$$

In the following, we will sketch how to solve the even perturbation. An observation is that, given some initial

\footnotetext{
${ }^{4}$ While this work was in progress, the interesting analysis of [40] appeared on the arXiv, also discussing the odd perturbation of stealth black holes but with further relaxing the assumptions to $A_{1} \neq 0$. Our results agree in the case of $A_{1}=0$.
}

conditions ${ }^{5}$ for $\delta \varphi$ and $h_{a b}^{\text {even }}$, one can directly solve for $\delta X$ as a whole from Eq. (3.7) (for a specific matter source distribution set by $\delta T_{a b}$ ). The expression for $\delta X$ can then be plugged into the effective source term $S_{a b}$ defined in Eq. (3.8), and this can then be used to solve for the remaining even-parity effective tensor mode in a very similar way as in GR. To see this work in practice, it is convenient to set a gauge and we do so differently

\footnotetext{
${ }^{5}$ Note that not all components in $h_{a b}^{\mathrm{even}}$ are independent as most of them are related constraint equations as we shall see later. To solve for the system, we only need to set initial conditions for $\chi, \dot{\chi}$, $\delta X$, and $\Phi$.
} 
depending on whether we are dealing with the monopole, dipole, or higher multipoles.

\section{Higher multipoles}

For multipoles with $\ell \geq 2$, we may fix the gauge by setting $\mathcal{G}=\mathcal{K}=\mathcal{H}_{0}=0$ and refer to Appendix A to see how coordinate transformations affect the even sector and check that this gauge can be chosen. The gauge fixing for monopole and dipole is different and will be treated separately below.

Having fixed $\mathcal{G}=\mathcal{K}=\mathcal{H}_{0}=0$, we can then derive explicitly the master equation for one of the propagating d.o.f. (effectively the even-parity tensor) by replacing $\mathrm{H}_{2}$ with a new variable $\chi$ defined through

$$
H_{2}=\frac{\ell(\ell+1)}{r} \mathcal{H}_{1}-\frac{1}{A r} \chi .
$$

For convenience, we denote

$$
\mathcal{E}_{a b}^{L} \equiv \delta R_{a b}-3 \Lambda h_{a b},
$$

so that Eq. (3.8) can be written as $\mathcal{E}_{a b}^{L}=S_{a b}$. The equation for $\chi$ can be obtained by considering the following combination:

$$
\begin{aligned}
\ddot{\chi} & A^{2} \chi_{, r r}+f_{1} \chi_{, r}+f_{2} \chi \\
\equiv & c_{1} \frac{\mathcal{E}_{t t}^{L}}{Y}+c_{2} \frac{\mathcal{E}_{r r}^{L}}{Y}+c_{3}\left(\frac{\mathcal{E}_{\theta \theta}^{L}}{Y}+\frac{\mathcal{E}_{\phi \phi}^{L}}{\sin ^{2} \theta Y}\right)+c_{4} \frac{\mathcal{E}_{r \theta}^{L}}{Y_{, \theta}} \\
& +c_{5} \frac{\mathcal{E}_{\theta \phi}^{L}}{\cot \theta Y_{, \phi}-Y_{, \theta \phi}}+d_{1} \frac{\mathcal{E}_{t t, r}^{L}}{Y}+d_{2} \frac{\mathcal{E}_{r r, r}^{L}}{Y} \\
& +d_{3}\left(\frac{\mathcal{E}_{\theta \theta}^{L}}{Y}+\frac{\mathcal{E}_{\phi \phi}^{L}}{\sin ^{2} \theta Y}\right)_{, r}+d_{4} \frac{\dot{\mathcal{E}}_{t r}^{L}}{Y},
\end{aligned}
$$

where $Y$ stands for the spherical harmonics, commas denote partial derivatives, and the coefficients $c_{i}, d_{i}$, and $f_{i}$ are given in Appendix B. Then the equation of motion for $\chi$ can be written as

$$
\ddot{\chi}-A^{2} \chi_{, r r}+f_{1} \chi_{, r}+f_{2} \chi=s(t, r),
$$

where $s(t, r)$ depends on $\delta X$ and $\delta T_{a b}$ and is given by the same combination as the right-hand side of Eq. (3.17) with $\mathcal{E}_{a b}^{L}$ replaced by $S_{a b}$. Using the following relations:

$$
\begin{gathered}
S_{t t}, S_{r r}, S_{t r} \propto Y, \\
F_{0} S_{r \theta}=F_{0}^{\prime} \delta X_{r \theta}+\delta T_{r \theta} \propto Y_{, \theta}, \\
F_{0} S_{\theta \phi}=F_{0}^{\prime} \delta X_{\theta \phi}+\delta T_{\theta \phi} \propto Y_{, \theta \phi}-\cot \theta Y_{, \phi}, \\
F_{0}\left(S_{\theta \theta}+\frac{S_{\phi \phi}}{\sin ^{2} \theta}\right) \propto Y,
\end{gathered}
$$

the angular dependence fully drops out from the right-hand side of Eq. (3.18).

In particular, the last relation (3.22) can be seen as follows. For $a, b=\theta, \phi$, terms in $S_{a b}$ proportional to $\bar{\varphi}_{a} \bar{\varphi}_{b}$ vanish, terms proportional to $\bar{g}_{a b}$ lead to contribution proportional to $r^{2} Y$, and terms involving covariant derivatives form the Laplacian operator in the two-sphere and therefore lead to contribution proportional to $\ell(\ell+1) Y$.

We can now (in principle) solve (3.18) explicitly for $\chi$ and infer the other components in the metric perturbations by considering the following constraint equations:

$$
\begin{aligned}
& \frac{r^{2}}{A} \frac{\mathcal{E}_{t t}^{L}}{Y}+A r^{2} \frac{\mathcal{E}_{r r}^{L}}{Y}+\left(\frac{\mathcal{E}_{\theta \theta}^{L}}{Y}+\frac{\mathcal{E}_{\phi \phi}^{L}}{\sin ^{2} \theta Y}\right) \\
& =-2 \chi, r-\frac{J}{r A} \chi-\frac{J\left(3 A+3 \Lambda r^{2}-J-1\right)}{r} \mathcal{H}_{1}, \\
& \frac{2 r^{2}}{J} \frac{\mathcal{E}_{t r}^{L}}{Y}=\dot{\mathcal{H}}_{1}-\frac{2}{J A} \dot{\chi}+H_{1}, \\
& \frac{2 \mathcal{E}_{\theta \phi}^{L}}{\cot \theta Y_{, \phi}-Y_{, \theta \phi}}=-2 A \mathcal{H}_{1, r}+\left(\frac{J}{2}-2 A_{, r}\right) \mathcal{H}_{1}-\frac{1}{r A} \chi-H_{0},
\end{aligned}
$$

where $J=\ell(\ell+1)$. Again, the above constraint equations are accompanied with some "source" terms on their righthand side, which are given by the same combination with $\mathcal{E}_{a b}^{L}$ replaced by $S_{a b}$, hence proving the constraints for $H_{0}$, $\mathcal{H}_{1}, H_{1}$ and $H_{2}$. With this in mind, we can then eventually solve the remaining dynamical d.o.f. (namely the scalar d.o.f.) $\Phi$ by using

$$
\begin{aligned}
\delta X= & {\left[\frac{2 q^{2} \sqrt{1-A}}{A} H_{1}+\frac{q^{2}(A-1)}{A} H_{2}-\frac{q^{2}}{A} H_{0}\right.} \\
& \left.+2 q \sqrt{1-A} \Phi_{, r}-\frac{2 q}{A} \dot{\Phi}\right] Y .
\end{aligned}
$$

The above analysis shows that, for multipoles with $\ell \geq 2$, there are two propagating d.o.f. in the even sector. With a trivial choice of $P, Q$, and $F$, we can get back to GR, in which case $\chi$ becomes the usual propagating d.o.f. in the even-parity sector. Therefore, we may think of the dynamical equations for the two d.o.f. in the even-parity sector as being Eqs. (3.7) and (3.18). We shall comment on this in what follows but first we look at the monopole and dipole.

\section{Monopole}

In the case of monopole, the contributions from $\mathcal{H}_{0}, \mathcal{H}_{1}$ and $\mathcal{G}$ vanish identically. We therefore instead set the gauge $\mathcal{K}=H_{1}=0$ (see Appendix A for confirmation that such a gauge can be fixed for the monopole). Then we find the following two constraints equations: 


$$
\begin{gathered}
\frac{r^{2}}{A} \frac{\mathcal{E}_{t t}^{L}}{Y}+A r^{2} \frac{\mathcal{E}_{r r}^{L}}{Y}+\left(\frac{\mathcal{E}_{\theta \theta}^{L}}{Y}+\frac{\mathcal{E}_{\phi \phi}^{L}}{\sin ^{2} \theta Y}\right) \\
=2 A r H_{2, r}+\left(2-6 \Lambda r^{2}\right) H_{2}, \\
\frac{r}{A^{2}} \frac{\mathcal{E}_{t t}^{L}}{Y}+r \frac{\mathcal{E}_{r r}^{L}}{Y}=H_{2, r}-H_{0, r},
\end{gathered}
$$

which confirms the fact that there are no monopole tensor modes and the relevant dynamics of the physical scalar monopole is given once again by Eq. (3.7).

\section{Dipole}

In the case of dipole, $h_{a b}^{\text {even }}$ only depends on $\mathcal{K}$ and $\mathcal{G}$ through the particular combination $\mathcal{K}-\mathcal{G}$, and thus we can set $\mathcal{K}=\mathcal{G}=\mathcal{H}_{0}=H_{2}=0$ by fixing gauge (see again Appendix A for confirmation that such a gauge can be fixed for the dipole). The other component can be solved by the constraint equations below.

$$
\begin{gathered}
\frac{r^{2}}{A} \frac{\mathcal{E}_{t t}^{L}}{Y}+A r^{2} \frac{\mathcal{E}_{r r}^{L}}{Y}+\left(\frac{\mathcal{E}_{\theta \theta}^{L}}{Y}+\frac{\mathcal{E}_{\phi \phi}^{L}}{\sin ^{2} \theta Y}\right) \\
=-4 A \mathcal{H}_{1, r}-\frac{2\left(1+A-3 \Lambda r^{2}\right)}{r} \mathcal{H}_{1}, \\
r^{2} \frac{\mathcal{E}_{t r}^{L}}{Y}=-\dot{\mathcal{H}}_{1}+H_{1}, \\
\frac{2 \mathcal{E}_{\theta \phi}^{L}}{\cot \theta Y_{, \phi}-Y_{, \theta \phi}}=-2 A \mathcal{H}_{1, r}-2 A_{, r} \mathcal{H}_{1}-H_{0},
\end{gathered}
$$

which also confirms the fact that there are no dipole tensor modes and the relevant dynamics of the physical scalar dipole is given also once again by Eq. (3.7).

\section{Dynamics of the scalar mode}

Whether we were dealing with the monopole, the dipole, or the higher multipoles, we have shown that the relevant dynamics for the physical scalar mode is governed solely by Eq. (3.7). With this in mind, we shall therefore focus on that equation more closely and instead of any of the gauge choices we used previously, we shall now set a gauge so that $\bar{\varphi}^{a} \bar{\varphi}^{b} h_{a b}^{\text {even }}=0$ irrespectively of which multipole we are dealing with. We emphasize that this is only for convenience, but none of the results depends on that precise gauge choice. In this case, it is easy to see that Eq. (3.7) becomes solely an equation for $\delta \varphi$ of the form

$$
\begin{gathered}
\bar{\varphi}^{a} \bar{\varphi}^{b} \mathrm{D}_{a} \mathrm{D}_{b} \delta \varphi+\left(\bar{\varphi}_{a} \bar{\varphi}^{a b}+\square \bar{\varphi} \bar{\varphi}^{b}+\frac{\bar{\varphi}^{a} \mathrm{D}_{a} \bar{\Omega}(r)}{\bar{\Omega}(r)} \bar{\varphi}^{b}\right) \partial_{b} \delta \varphi \\
=\frac{1}{\bar{\Omega}(r)} D_{a}\left(\frac{F_{0}^{\prime}}{F_{0}} \bar{\varphi}^{a} \delta T\right),
\end{gathered}
$$

where $\bar{\Omega}(r)=12 \Lambda F_{0}^{\prime \prime}+P_{0}^{\prime \prime}+Q_{0}^{\prime \prime} \square \bar{\varphi}+12 \Lambda F_{0}^{\prime 2} / F_{0}$. Given the background solution, we see that the scalar fluctuation $\delta \varphi$ sees a singular effective metric $g_{\mathrm{eff}}^{a b} \sim \bar{\varphi}^{a} \bar{\varphi}^{b}$, which only ever has one nonvanishing eigenvalue. This implies that the physical (diagonalized) scalar fluctuations living on this exact black hole solution would be thus infinitely strongly coupled and the background solution cannot be trusted.

The strong coupling issue can be understood as follows. Consider, for instance, the EFT of scalar field $\pi$ with operators that enter at an arbitrary scale $\Lambda$ (that could potentially be as large as the Planck Scale),

$$
S_{\text {toy }}=\int \mathrm{d} t \mathrm{~d}^{3} x\left[\frac{1}{2}\left(\partial_{t} \pi\right)^{2}-\frac{B}{2}(\nabla \pi)^{2}+\frac{(\nabla \pi)^{3}}{\Lambda^{2}}\right] .
$$

In this model, just like in the theory we have considered in this paper, the effective metric of $\pi$ becomes singular as $B \rightarrow 0$. The strong coupling problem is always present when $B \rightarrow 0$, but becomes manifest after performing the following redefinitions so that the kinetic term is canonical:

$$
t \rightarrow t / \sqrt{B} \text { and } \pi \rightarrow \pi / B^{1 / 4}
$$

In these new variables, the action (3.33) is

$$
S_{\text {toy }}=\int \mathrm{d} t \mathrm{~d}^{3} x\left[\frac{1}{2}\left(\partial_{t} \pi\right)^{2}-\frac{1}{2}(\nabla \pi)^{2}+\frac{1}{B^{5 / 4}} \frac{(\nabla \pi)^{3}}{\Lambda^{2}}\right] .
$$

As $B \rightarrow 0$, it is now manifest that the toy model becomes infinitely strongly coupled and there is no sense in which perturbations theories can be trusted. This means that the background solution itself cannot be trusted for these types of models. This is precisely the issue that arises in the stealth black hole solutions considered here. While the example considered here is simply that of a scalar field, the argument applies to any nonlinear relativistic field theory as is the case for any gravitational theory. Being a gravitational theory, the DHOST models considered here involve an infinite number of nonlinear interactions. In the theory at hand, perturbing the action (2.5) around the black hole solution leads to nonlinear interactions including terms of the form $P_{0}^{\prime \prime} \bar{\varphi}^{b} \delta \varphi_{b} \delta \varphi^{a} \delta \varphi_{a}, P_{0}^{\prime \prime \prime} \bar{\varphi}^{b} \delta \varphi_{b}\left(\delta \varphi^{a} \delta \varphi_{a}\right)^{2}$, etc., involving spatial derivatives acting on the scalar d.o.f. As we have seen in the toy model, a singular effective metric implies strong couplings of these nonlinear interactions and hence the breakdown of perturbation theory. One may avoid the strong coupling issue by setting all the derivatives of $P, Q, F$, and $A_{i}$ to vanish to all orders. This would then lead to a trivial scalar field theory which would not enjoy the stealth black hole solution considered here. Notice that it is the fundamental relativistic structure of the theory which enforces the existence of operators with spatial derivatives of the form (3.33). Had we been dealing with a nonrelativistic theory or what that manifestly breaks Lorentz invariance, the existence of spatial derivative in the 
nonlinear interactions could have been avoided and strong coupling would be prevented. However, for theories such as Horndeski, DHOST or other relativistic theories, singularity of the effective metric leads to strong coupling. We refer to $[53,60]$ for further discussions on the issue of strong coupling in these types of theories.

\section{STRONG COUPLING ISSUES FOR GENERIC MANIFOLDS}

Quadratic DHOST: Before concluding, it is worth pointing out that the strong coupling results derived in this paper hold beyond the SdS metric considered here and are actually generalizable to much more generic manifolds ${ }^{6}$ and scenarios so long as $X$ is constant on the background manifold, $X=X_{0}=$ const.

Indeed, consider the full quadratic DHOST theory (2.1) with generic shift-symmetric functions $P, F, Q$, and $A_{i}(X)$, then we can show that any background solution (denoted by the subscript ${ }_{0}$ ) that satisfies the following properties on that particular background solution suffers from infinitely strong coupling and cannot be trusted,

$$
\left\{\begin{array}{l}
X_{0}=\text { const, } \\
A_{1}\left(X_{0}\right)=A_{2}\left(X_{0}\right)=A_{1}^{\prime}\left(X_{0}\right)=A_{2}^{\prime}\left(X_{0}\right)=0, \\
A_{3}\left(X_{0}\right)=A_{5}\left(X_{0}\right)=Q^{\prime}\left(X_{0}\right)=0, \\
A_{4}\left(X_{0}\right)=6 \frac{F^{\prime}\left(X_{0}\right)^{2}}{F\left(X_{0}\right)}, \\
P^{\prime}\left(X_{0}\right)=-R_{0} F^{\prime}\left(X_{0}\right) .
\end{array}\right.
$$

This can be seen by considering

$$
\mathcal{E}_{\varphi}^{(1)}-\frac{2 F_{0}^{\prime}}{F_{0}-X_{0} F_{0}^{\prime}} \square \bar{\varphi} \mathcal{E}^{(1)}-\frac{2 F_{0}^{\prime}}{F_{0}-X_{0} F_{0}^{\prime}} \mathrm{D}_{a}\left[\mathcal{E}^{(1)} \bar{\varphi}^{a}\right]=0,
$$

where $\mathcal{E}_{\varphi}^{(1)}$ and $\mathcal{E}_{a b}^{(1)}$ are the field equation and the modified Einstein equation derived from the full quadratic DHOST theory (2.1), and $\mathcal{E}^{(1)}=\bar{g}^{a b} \mathcal{E}_{a b}^{(1)}$. Subject to condition (4.1), Eq. (4.2) is solely an equation for $\delta \varphi$ with the highest order derivative proportional to $\bar{\varphi}^{a} \bar{\varphi}^{b} \mathrm{D}_{a} \mathrm{D}_{b} \delta \varphi$. In particular, this implies that the rotating black hole solutions found in [61] suffer from the same issue, and fluctuations of the scalar d.o.f. about the rotating black hole found in [61] with finite stealth hair are infinitely strongly coupled (apart in the special case of spherical symmetry where the constraint $A_{3}\left(X_{0}\right)=0$ is relaxed).

Note that those conditions do not impose to be dealing with theories where for instance $A_{3}, A_{5}$, and $Q^{\prime}$ vanish identically, and strong coupling would still be an issue even if say $A_{3}^{\prime}\left(X_{0}\right) \neq 0$ or $A_{5}^{\prime}\left(X_{0}\right) \neq 0$ or $A_{1}^{\prime \prime}\left(X_{0}\right) \neq 0$. To avoid strong coupling, at least one of the constraints in (4.1)

\footnotetext{
${ }^{6}$ We wish to thank Hayato Motohashi for very useful discussion on this point.
}

should be violated but note also that the conditions (4.1) are not the unique conditions under which the issue may arise, and violating one or several of the conditions in (4.1) does not necessarily ensure the absence of strong coupling issue. It is possible that strong coupling occurs on particular solutions even if the previous conditions are not satisfied, or that strong coupling arises instead for the tensor d.o.f. [11].

Further relaxing the assumptions: One may raise the question of what would occur if for instance $A_{3}\left(X_{0}\right)$ did not vanish precisely but was simply taken to be (extremely) small so as to prevent too much GW decay into dark energy on a particular solution of interest (of course if the EFT is not valid on those scales, the constraint on $A_{3}$ could potentially be relaxed further). If, for instance, $A_{3}\left(X_{0}\right)$ was considered to be small but nonvanishing, then the effective metric of the scalar d.o.f. about that solution could include four nonvanishing eigenvalues but the magnitude of those would be governed by the (extremely) small scale present in $A_{3}\left(X_{0}\right)$ and would also indicate strong coupling issues (low cutoff). For instance, if one were to consider perturbations about a spherically symmetric configuration, this would imply that the higher multipoles would not be suppressed as compared to lower multipoles. Second, since $\delta \varphi$ does couple to the trace of external matter fields in generic DHOST theories, as can be seen from the righthand side of (3.32), any small test particle would lead to arbitrarily large emission of scalar waves. ${ }^{7}$

Horndeski: This also applies to any Horndeski theories [1] that satisfies an equivalent set of conditions, independently of how symmetric (or not) the background manifold is. Consider a shift-symmetric Horndeski theory of the form

$$
\begin{aligned}
S_{\text {Horndeski }}= & \int \mathrm{d}^{4} x \sqrt{-g}\left[K(X)-G_{3}(X) \square \varphi-2 G_{4}(X) R\right. \\
& +G_{4}^{\prime}(X)\left((\square \varphi)^{2}-\varphi_{a b}^{2}\right)+G_{5}(X) G_{a b} \varphi^{a} \varphi^{b} \\
& \left.+\frac{1}{3} G_{5}^{\prime}(X)\left((\square \varphi)^{3}-3 \square \varphi \varphi_{a b}^{2}+2 \varphi_{a b}^{3}\right)\right], \quad
\end{aligned}
$$

with minimal coupling to external sources. Then for this theory, any solution on which $X_{0}$ is constant and for which $G_{3}^{\prime}\left(X_{0}\right)=G_{4}^{\prime \prime}\left(X_{0}\right)=G_{5}^{\prime}\left(X_{0}\right)=G_{5}^{\prime \prime}\left(X_{0}\right)=K^{\prime}\left(X_{0}\right)=0$,

the scalar propagating d.o.f. about this would be solution is infinitely strongly coupled and the existence of such a solution could not be trusted. This result is independent of any details of the manifold considered and the symmetry of the solution. This is in addition to potential strong coupling issue that may occur for the tensor modes.

\footnotetext{
${ }^{7}$ Unless $F_{0}^{\prime}=0$ in which case (2.9) or (2.10) would also imply $Q_{0}^{\prime}=P_{0}^{\prime}=0$ and we would have $A_{4}\left(X_{0}\right)=0$, then on that background $P_{0}$ would effectively play the role of a cosmological constant, the term proportional to $Q_{0}$ would be a total derivative and we would effectively just be dealing with GR and a scalar field minimally coupled to gravity.
} 
In particular, we may point out that for appropriate choices of functions $A_{i}$, the DHOST theory considered in (2.1) reduces to a special case of Horndeski [1], for which the static black hole solutions and their stability were explored in [62] and the strong coupling results remain valid in that particular subclass of Horndeski.

Indeed, following the analysis performed in [62] (which applied for static solutions $q=0)$, with $G_{3, X}\left(X_{0}\right)=$ $K_{, X}\left(X_{0}\right)=0, G_{4}$ to be constant and imposing $G_{5}$ to vanish we find that the effective metric for the dynamical evend.o.f. is singular, see Appendix C, confirming a strong coupling issue for that particular limit of the Horndeski black Hole solutions. In that case, this strong coupling issue appears to be closely linked to the requirement that $X$ be a constant at the background level.

\section{OUTLOOKS}

In this paper, we investigated the perturbation of linearly time-dependent stealth SdS black holes in shift-symmetric quadratic DHOST theories. We focus on the subclass of DHOST theories described by action (2.5), i.e., those theories that (1) predict unitary GW speed and (2) have no significant decay of GWs into the scalar fluctuations. The linearly time-dependent stealth SdS black holes exist if the functions in action (2.5) satisfy condition (2.9) or (2.10). As usual, we decomposed the perturbation based on their parity and derived the perturbation equations, respectively.

By deriving the perturbation equations, we find that the odd-parity perturbation is the same as that of GR black holes. Actually, this is the case even if the DHOST theories involve a nontrivial $A_{3}$ (in which case the stealth SdS black holes also exist). Since the background geometry is exactly $\mathrm{SdS}$, the scalar perturbation does not couple with the oddparity metric perturbation. We also find that the even-parity perturbations are different from that in GR in general. The metric perturbation could be sourced by the perturbation of external matter field in a different way due to the presence of the scalar field. More concerning, we find that the scalar fluctuation sees a singular effective metric and hence suffers from a strong coupling problem. The black hole solution considered is therefore beyond the regime of validity of the DHOST effective field theory and cannot be trusted.

Finally, we point out that the issue of strong coupling derived in this paper is very generic to a large class of DHOST and Horndeski solutions. In particular, those issues apply to other rotating black hole solutions with scalar hair found in the literature and in some subclasses of Horndeski theories. We show that under a set of conditions DHOST and Horndeski solutions suffer the same scalar strong coupling issue irrespectively of the specific manifold and symmetry of the system.

\section{ACKNOWLEDGMENTS}

We would like to thank Christos Charmousis, Hayato Motohashi, and Huan Yang for helpful discussion and suggestions. C.d.R. would like thank the Perimeter Institute for Theoretical Physics for its hospitality during part of this work and for support from the Simons Emmy Noether program. The work of C. d. R. is supported by an STFC Grant No. ST/P000762/1. C. d. R. thanks the Royal Society for support at ICL through a Wolfson Research Merit Award. C.d. R. and J.Z. are supported by the European Union's Horizon 2020 Research Council Grant No. 724659 MassiveCosmo ERC-2016-COG. C. d. R. is also supported by a Simons Foundation Award No. 555326 under the Simons Foundation's Origins of the Universe initiative, Cosmology Beyond Einstein's Theory.

\section{APPENDIX A: EVEN-PARITY COORDINATE TRANSFORMATIONS}

In this appendix, we shall see the effect of an even-parity coordinate transformation so as to motivate our gauge chosen in the study of the even sector of Sec. III B. Recalling that the even-parity metric perturbations can be written as

$$
h_{a b}^{\text {even }}=\left(\begin{array}{cccc}
A H_{0} & H_{1} & \mathcal{H}_{0} \partial_{\theta} & \mathcal{H}_{0} \partial_{\phi} \\
H_{1} & H_{2} / A & \mathcal{H}_{1} \partial_{\theta} & \mathcal{H}_{1} \partial_{\phi} \\
\mathcal{H}_{0} \partial_{\theta} & \mathcal{H}_{1} \partial_{\theta} & \mathcal{K}+\mathcal{G} \nabla_{\theta} \nabla_{\theta} & \mathcal{G} \nabla_{\theta} \nabla_{\phi} \\
\mathcal{H}_{0} \partial_{\phi} & \mathcal{H}_{1} \partial_{\phi} & \mathcal{G} \nabla_{\phi} \nabla_{\theta} & \sin ^{2} \theta \mathcal{K}+\mathcal{G} \nabla_{\phi} \nabla_{\phi}
\end{array}\right) Y_{\text {lm }}
$$

and the scalar perturbation as

$$
\delta \varphi=\Phi(t, r) Y_{\ell m},
$$

we now consider an infinitesimal coordinate transformation $x^{a} \rightarrow \tilde{x}^{a}=x^{a}+\xi^{a}$ with

$$
\xi^{a}=\left(\mathrm{T}(t, r), \mathcal{R}(t, r), \Theta(t, r) \partial_{\theta}, \frac{\Theta(t, r) \partial_{\phi}}{\sin ^{2} \theta}\right) Y_{\ell m}(\theta, \phi)
$$


Then the metric perturbations in Eq. (A1) transform as follows:

$$
\begin{gathered}
H_{0} \rightarrow \tilde{H}_{0}=H_{0}+2 \dot{T}+\frac{A_{, r}}{A} \mathcal{R}, \\
H_{1} \rightarrow \tilde{H}_{1}=H_{1}+A \mathrm{~T}_{, r}-\dot{\mathcal{R}} / A, \\
H_{2} \rightarrow \tilde{H}_{2}=H_{2}+\frac{A_{, r}}{A} \mathcal{R}-2 \mathcal{R}_{, r}, \\
\mathcal{H}_{0} \rightarrow \tilde{\mathcal{H}}_{0}=\mathcal{H}_{0}+A \mathrm{~T}-r^{2} \dot{\Theta}, \\
\mathcal{H}_{1} \rightarrow \tilde{\mathcal{H}}_{1}=\mathcal{H}_{1}-\mathcal{R} / A-r^{2} \Theta_{, r}, \\
\mathcal{G} \rightarrow \tilde{\mathcal{G}}=\mathcal{G}-2 \Theta, \\
\mathcal{K} \rightarrow \tilde{\mathcal{K}}=\mathcal{K}-\frac{2}{r} \mathcal{R} .
\end{gathered}
$$

For multipoles $\ell \geq 2$, one can set the gauge $\tilde{\mathcal{G}}=\tilde{\mathcal{K}}=$ $\tilde{\mathcal{H}}_{0}=0$ by an appropriate choice of the respective functions $\Theta, \mathcal{R}$, and $\mathrm{T}$ and can omit the tildes from now on.

For monopole, Eq. (A1) becomes

$\left.h_{a b}^{\mathrm{even}}\right|_{\ell=0}=\frac{1}{2 \sqrt{\pi}}\left(\begin{array}{cccc}A H_{0} & H_{1} & 0 & 0 \\ H_{1} & H_{2} / A & 0 & 0 \\ 0 & 0 & \mathcal{K} & 0 \\ 0 & 0 & 0 & \sin ^{2} \theta \mathcal{K}\end{array}\right)$,

while the gauge transformation (A3) involves two free functions $T$ and $\mathcal{R}$, which can be chosen appropriately so as to fix $\mathcal{K}=H_{1}=0$.

Finally, for dipole, Eq. (A1) becomes

$$
\begin{aligned}
& \left.h_{a b}^{\text {even }}\right|_{\ell=1} \\
& =\left(\begin{array}{cccc}
A H_{0} & H_{1} & \mathcal{H}_{0} \partial_{\theta} & \mathcal{H}_{0} \partial_{\phi} \\
H_{1} & H_{2} / A & \mathcal{H}_{1} \partial_{\theta} & \mathcal{H}_{1} \partial_{\phi} \\
\mathcal{H}_{0} \partial_{\theta} & \mathcal{H}_{1} \partial_{\theta} & \mathcal{K}-\mathcal{G} & 0 \\
\mathcal{H}_{0} \partial_{\phi} & \mathcal{H}_{1} \partial_{\phi} & 0 & \sin ^{2} \theta(\mathcal{K}-\mathcal{G})
\end{array}\right) Y_{1 m},
\end{aligned}
$$

which depends on $\mathcal{K}$ and $\mathcal{G}$ only through $\mathcal{K}-\mathcal{G}$. The gauge transformation (A3) still involves three free functions $\Theta, T$, and $\mathcal{R}$, which can be chosen so as to set $\mathcal{K}-\mathcal{G}=\mathcal{H}_{0}=H_{2}=0$.

\section{APPENDIX B: EXPRESSIONS OF THE COEFFICIENTS}

The coefficients in Eq. (3.17) are defined as follows:

$$
\begin{gathered}
c_{1}=\frac{\left(4+J-6 A-6 \Lambda r^{2}\right) A r}{1+J-3 A-3 \Lambda r^{2}}, \\
c_{2}=-\frac{\left[3 A^{2}-6 \Lambda A r^{2}+\left(1+J-3 \Lambda r^{2}\right)\left(3 \Lambda r^{2}-1\right)\right] A^{2} r}{1+J-3 A-3 \Lambda r^{2}}, \\
c_{3}=-\frac{\left[J^{2}+3 A^{2}-2 A\left(1+J+3 \Lambda r^{2}\right)-\left(1-3 \Lambda r^{2}\right)^{2}\right] A}{2 r\left(1+J-3 A-3 \Lambda r^{2}\right)}, \\
c_{4}=J A^{2}, \\
c_{5}=\frac{J A\left(J-2 A+r A_{, r}\right)}{2 r},
\end{gathered}
$$

$d_{1}=\frac{1}{2} A r^{2}, \quad d_{2}=\frac{1}{2} A^{3} r^{2}, \quad d_{3}=\frac{1}{2} A^{2}, \quad d_{4}=-A r^{2}$,

$$
\begin{gathered}
f_{1}=\frac{A\left[3 A^{2}+A\left(J-2-6 \Lambda r^{2}\right)-\left(J+1-3 \Lambda r^{2}\right)\left(1-3 \Lambda r^{2}\right)\right]}{r\left(J+1-3 A-3 \Lambda r^{2}\right)}, \\
f_{2}=\frac{(J-2) J A}{r^{2}\left(J+1-3 A-3 \Lambda r^{2}\right)},
\end{gathered}
$$

where $J=\ell(\ell+1)$.

\section{APPENDIX C: HORNDESKI BLACK HOLE SOLUTIONS}

Perturbations about static black hole solutions in Horndeski (4.3) were explored in [62]. While the analysis performed in the paper applied to DHOST theories, one can show that they would be applicable to the special subclass of solutions explored in [62] when $q=0$ and when taking $G_{3, X}\left(X_{0}\right)=K_{, X}\left(X_{0}\right)=0$, while keeping $G_{4}$ to be constant, $G_{4}(X)=\bar{G}_{4}=$ const and imposing $G_{5}$ to vanish identically.

Upon these restrictions, one can confirm that the variable $\Sigma$ defined in Eq. (36) of [62] vanishes and the variable $\mathcal{P}_{1}$ defined in Eq. (34) is given by $\mathcal{P}_{1}=\bar{G}_{4}=\frac{1}{2} \mathcal{F}$, hence implying that the dynamical metric $\mathcal{K}$ is always singular for that subclass of solutions $\operatorname{det}(\mathcal{K})=0$ as can be seen from Eq. (38) of [62], in agreement with the results presented here. 
[1] G. W. Horndeski, Second-order scalar-tensor field equations in a four-dimensional space, Int. J. Theor. Phys. 10, 363 (1974).

[2] C. Deffayet, X. Gao, D. A. Steer, and G. Zahariade, From k-essence to generalized Galileons, Phys. Rev. D 84, 064039 (2011).

[3] C. de Rham, G. Gabadadze, and A. J. Tolley, Ghost free massive gravity in the Stúckelberg language, Phys. Lett. B 711, 190 (2012).

[4] C. de Rham, G. Gabadadze, and A. J. Tolley, Helicity decomposition of ghost-free massive gravity, J. High Energy Phys. 11 (2011) 093.

[5] D. Langlois and K. Noui, Degenerate higher derivative theories beyond Horndeski: Evading the Ostrogradski instability, J. Cosmol. Astropart. Phys. 02 (2016) 034.

[6] M. Zumalacrregui and J. Garca-Bellido, Transforming gravity: From derivative couplings to matter to secondorder scalar-tensor theories beyond the Horndeski Lagrangian, Phys. Rev. D 89, 064046 (2014).

[7] H. Motohashi and T. Suyama, Third order equations of motion and the Ostrogradsky instability, Phys. Rev. D 91, 085009 (2015).

[8] J. Ben Achour, M. Crisostomi, K. Koyama, D. Langlois, K. Noui, and G. Tasinato, Degenerate higher order scalartensor theories beyond Horndeski up to cubic order, J. High Energy Phys. 12 (2016) 100.

[9] J. Ben Achour, D. Langlois, and K. Noui, Degenerate higher order scalar-tensor theories beyond Horndeski and disformal transformations, Phys. Rev. D 93, 124005 (2016).

[10] M. Crisostomi, K. Koyama, and G. Tasinato, Extended scalar-tensor theories of gravity, J. Cosmol. Astropart. Phys. 04 (2016) 044.

[11] C. de Rham and A. Matas, Ostrogradsky in theories with multiple fields, J. Cosmol. Astropart. Phys. 06 (2016) 041.

[12] H. Motohashi, K. Noui, T. Suyama, M. Yamaguchi, and D. Langlois, Healthy degenerate theories with higher derivatives, J. Cosmol. Astropart. Phys. 07 (2016) 033.

[13] D. Langlois, Dark energy and modified gravity in degenerate higher-order scalartensor (DHOST) theories: A review, Int. J. Mod. Phys. D 28, 1942006 (2019).

[14] B. P. Abbott et al. (LIGO Scientific and Virgo Collaborations), GW170817: Observation of Gravitational Waves from a Binary Neutron Star Inspiral, Phys. Rev. Lett. 119, 161101 (2017).

[15] A. Goldstein et al., An ordinary short $\gamma$-ray burst with extraordinary implications: Fermi-GBM detection of GRB 170817A, Astrophys. J. 848, L14 (2017).

[16] B. P. Abbott et al. (LIGO Scientific, Virgo, Fermi-GBM, and INTEGRAL Collaboration), Gravitational waves and $\gamma$-rays from a binary neutron star merger: GW170817 and GRB 170817A, Astrophys. J. 848, L13 (2017).

[17] P. Creminelli and F. Vernizzi, Dark Energy after GW170817 and GRB170817A, Phys. Rev. Lett. 119, 251302 (2017).

[18] J. Sakstein and B. Jain, Implications of the Neutron Star Merger GW170817 for Cosmological Scalar-Tensor Theories, Phys. Rev. Lett. 119, 251303 (2017).

[19] J. M. Ezquiaga and M. Zumalacàrregui, Dark Energy After GW170817: Dead Ends and the Road Ahead, Phys. Rev. Lett. 119, 251304 (2017).
[20] T. Baker, E. Bellini, P. G. Ferreira, M. Lagos, J. Noller, and I. Sawicki, Strong Constraints on Cosmological Gravity from GW170817 and GRB 170817A, Phys. Rev. Lett. 119, 251301 (2017).

[21] D. Langlois, R. Saito, D. Yamauchi, and K. Noui, Scalartensor theories and modified gravity in the wake of GW170817, Phys. Rev. D 97, 061501 (2018).

[22] C. de Rham and S. Melville, Gravitational Rainbows: LIGO and Dark Energy at its Cutoff, Phys. Rev. Lett. 121, 221101 (2018).

[23] L. Hui and A. Nicolis, No-Hair Theorem for the Galileon, Phys. Rev. Lett. 110, 241104 (2013).

[24] E. Babichev, C. Charmousis, and A. Lehbel, Asymptotically flat black holes in Horndeski theory and beyond, J. Cosmol. Astropart. Phys. 04 (2017) 027.

[25] T. P. Sotiriou and S.-Y. Zhou, Black Hole Hair in Generalized Scalar-Tensor Gravity, Phys. Rev. Lett. 112, 251102 (2014).

[26] T. P. Sotiriou and S.-Y. Zhou, Black hole hair in generalized scalar-tensor gravity: An explicit example, Phys. Rev. D 90, 124063 (2014).

[27] G. Antoniou, A. Bakopoulos, and P. Kanti, Evasion of No-Hair Theorems and Novel Black-Hole Solutions in Gauss-Bonnet Theories, Phys. Rev. Lett. 120, 131102 (2018).

[28] G. Antoniou, A. Bakopoulos, and P. Kanti, Black-hole solutions with scalar hair in Einstein-Scalar-Gauss-Bonnet theories, Phys. Rev. D 97, 084037 (2018).

[29] A. Bakopoulos, G. Antoniou, and P. Kanti, Novel blackhole solutions in Einstein-Scalar-Gauss-Bonnet theories with a cosmological constant, Phys. Rev. D 99, 064003 (2019).

[30] L. Berezhiani, G. Chkareuli, C. de Rham, G. Gabadadze, and A. J. Tolley, On black holes in massive gravity, Phys. Rev. D 85, 044024 (2012).

[31] R. A. Rosen, Non-singular black holes in massive gravity: Time-dependent solutions, J. High Energy Phys. 10 (2017) 206.

[32] C. de Rham, G. Gabadadze, and A. J. Tolley, Resummation of Massive Gravity, Phys. Rev. Lett. 106, 231101 (2011).

[33] E. Babichev and C. Charmousis, Dressing a black hole with a time-dependent Galileon, J. High Energy Phys. 08 (2014) 106.

[34] C. Charmousis, T. Kolyvaris, E. Papantonopoulos, and M. Tsoukalas, Black holes in bi-scalar extensions of Horndeski theories, J. High Energy Phys. 07 (2014) 085.

[35] E. Babichev and G. Esposito-Farse, Time-dependent spherically symmetric covariant Galileons, Phys. Rev. D 87, 044032 (2013).

[36] T. Kobayashi and N. Tanahashi, Exact black hole solutions in shift symmetric scalartensor theories, Prog. Theor. Exp. Phys. 2014, $73 \mathrm{E} 02$ (2014).

[37] E. Babichev and G. Esposito-Farese, Cosmological self-tuning and local solutions in generalized Horndeski theories, Phys. Rev. D 95, 024020 (2017).

[38] H. Motohashi and M. Minamitsuji, General relativity solutions in modified gravity, Phys. Lett. B 781, 728 (2018).

[39] M. Minamitsuji and H. Motohashi, Stealth Schwarzschild solution in shift symmetry breaking theories, Phys. Rev. D 98, 084027 (2018). 
[40] K. Takahashi, H. Motohashi, and M. Minamitsuji, Linear stability analysis of hairy black holes in quadratic degenerate higher-order scalar-tensor theories: Odd-parity perturbations, Phys. Rev. D 100, 024041 (2019).

[41] E. Ayon-Beato, C. Martinez, and J. Zanelli, Stealth scalar field overflying a $(2+1)$ black hole, Gen. Relativ. Gravit. 38, 145 (2006).

[42] J. Ben Achour and H. Liu, Hairy Schwarzschild-(A)dS black hole solutions in degenerate higher order scalar-tensor theories beyond shift symmetry, Phys. Rev. D 99, 064042 (2019).

[43] H. Motohashi and M. Minamitsuji, Exact black hole solutions in shift-symmetric quadratic degenerate higherorder scalar-tensor theories, Phys. Rev. D 99, 064040 (2019).

[44] A. Silvestri, Scalar Radiation from Chameleon-Shielded Regions, Phys. Rev. Lett. 106, 251101 (2011).

[45] C. de Rham, A. J. Tolley, and D. H. Wesley, Vainshtein mechanism in binary pulsars, Phys. Rev. D 87, 044025 (2013).

[46] C. de Rham, A. Matas, and A. J. Tolley, Galileon radiation from binary systems, Phys. Rev. D 87, 064024 (2013).

[47] F. Dar, C. De Rham, J. T. Deskins, J. T. Giblin, and A. J. Tolley, Scalar gravitational radiation from binaries: Vainshtein mechanism in time-dependent systems, Classical Quantum Gravity 36, 025008 (2019).

[48] L. Sagunski, J. Zhang, M. C. Johnson, L. Lehner, M. Sakellariadou, S. L. Liebling, C. Palenzuela, and D. Neilsen, Neutron star mergers as a probe of modifications of general relativity with finite-range scalar forces, Phys. Rev. D 97, 064016 (2018).

[49] J. Huang, M. C. Johnson, L. Sagunski, M. Sakellariadou, and J. Zhang, Prospects for axion searches with Advanced LIGO through binary mergers, Phys. Rev. D 99, 063013 (2019).

[50] H. Ogawa, T. Kobayashi, and T. Suyama, Instability of hairy black holes in shift-symmetric Horndeski theories, Phys. Rev. D 93, 064078 (2016).
[51] E. Babichev, C. Charmousis, G. Esposito-Farse, and A. Lehbel, Stability of Black Holes and the Speed of Gravitational Waves within Self-Tuning Cosmological Models, Phys. Rev. Lett. 120, 241101 (2018).

[52] E. Babichev, C. Charmousis, G. Esposito-Farse, and A. Lehbel, Hamiltonian unboundedness vs stability with an application to Horndeski theory, Phys. Rev. D 98, 104050 (2018).

[53] C. de Rham and S. Melville, Unitary null energy condition violation in $\mathrm{P}(\mathrm{X})$ cosmologies, Phys. Rev. D 95, 123523 (2017).

[54] G. D'Amico, C. de Rham, S. Dubovsky, G. Gabadadze, D. Pirtskhalava, and A. J. Tolley, Massive cosmologies, Phys. Rev. D 84, 124046 (2011).

[55] P. Creminelli, M. Lewandowski, G. Tambalo, and F. Vernizzi, Gravitational wave decay into dark energy, J. Cosmol. Astropart. Phys. 12 (2018) 025.

[56] P. Creminelli, G. Tambalo, F. Vernizzi, and V. Yingcharoenrat, Resonant decay of gravitational waves into dark energy, J. Cosmol. Astropart. Phys. 10 (2019) 072.

[57] T. Regge and J. A. Wheeler, Stability of a schwarzschild singularity, Phys. Rev. 108, 1063 (1957).

[58] F. J. Zerilli, Effective Potential for Even Parity ReggeWheeler Gravitational Perturbation Equations, Phys. Rev. Lett. 24, 737 (1970).

[59] H. Motohashi and T. Suyama, Black hole perturbation in parity violating gravitational theories, Phys. Rev. D 84, 084041 (2011).

[60] L. Berezhiani, G. Chkareuli, C. de Rham, G. Gabadadze, and A. J. Tolley, Mixed Galileons and spherically symmetric solutions, Classical Quantum Gravity 30, 184003 (2013).

[61] C. Charmousis, M. Crisostomi, R. Gregory, and N. Stergioulas, Rotating black holes in higher order gravity, Phys. Rev. D 100, 084020 (2019).

[62] T. Kobayashi, H. Motohashi, and T. Suyama, Black hole perturbation in the most general scalar-tensor theory with second-order field equations II: The even-parity sector, Phys. Rev. D 89, 084042 (2014). 